\title{
AN ASYMPTOTIC EXPANSION FOR THE TAIL OF COMPOUND SUMS OF BURR DISTRIBUTED RANDOM VARIABLES*
}

\author{
Dominik Kortschak Hansjörg Albrecher \\ Department of Actuarial Science, Faculty of Business and Economics, University of Lausanne \\ Batiment Extranef, UNIL-Dorigny, 1015 Lausanne, Switzerland
}

\begin{abstract}
In this paper we show that it is possible to write the Laplace transform of the Burr distribution as the sum of four series. This representation is then used to provide a complete asymptotic expansion of the tail of the compound sum of Burr distributed random variables. Furthermore it is shown that if the number of summands is fixed, this asymptotic expansion is actually a series expansion if evaluated at sufficiently large arguments.
\end{abstract}

Keywords: Compound sum, Burr distribution, asymptotic expansion, Laplace transform.

\section{Introduction}

Consider the compound sum $S_{N}=X_{1}+\ldots+X_{N}$, where $\left(X_{i}\right)_{i \geq 1}$ is a sequence of independent and identically distributed random variables with distribution function $F$ and $N$ is an integer-valued random variable with probability function $\mathbb{P}(N=n)=p_{n}$ (independent of the $\left.X_{i}\right)$. The determination of approximations for the tail probability

$$
\bar{G}(u):=\mathbb{P}\left(S_{N}>u\right) .
$$

is a classical problem in applied probability. It occurs for instance in risk theory as the probability that the total claim amount in the collective risk model exceeds a certain threshold (cf. Klugman et al. (2008)) or in various applications in queueing theory (cf. Asmussen (2003)). Also, the probability of the maximum of a random walk with negative drift to overshoot some given level can be expressed in the above way in a variety of models (cf. Rolski et al. (1999)). For subexponential distribution functions $F$, a first-order asymptotic approximation is provided in e.g. Embrechts and Veraverbeke (1982). Under certain further conditions on $F$, higher-order asymptotic expansions have been derived, see e.g. Grübel (1987), Baltrūnas and Omey (1998), Borovkov and Borovkov (2002), Barbe and McCormick (2005, 2009); Barbe et al. (2007), Geluk (1992, 1996); Geluk et al. (2000) and Albrecher et al. (2010). All these results have the drawback that, for regularly varying distributions, the asymptotic expansion ends after a finite number of terms. On the other hand, in Brennan et al. (1968) an infinite order expansion for $\bar{G}(u)$ was provided

\footnotetext{
*Supported by the Austrian Science Foundation Project P18392-MAT and the Swiss National Science Foundation Project 200021-124635/1.
} 
for the special case of Pareto summands with $\bar{F}(x)=1-F(x)=x^{-\alpha}$ and deterministic $N=n$ (Blum (1970) later showed that this expansion converges and equality holds). The main tool there was to invert the Laplace transform of the Pareto distribution, which contains an incomplete Gamma function, and its available series expansion. In Albrecher and Kortschak (2009) an infinite order expansion for $\bar{G}(u)$ was identified (in the context of ruin probabilities) for a geometric compound sum with summands whose integrated tail is Pareto distributed. From Section 4 of Albrecher and Kortschak (2009) it becomes clear that this procedure can also be generalized to other compounding distributions.

In this paper we will show that if the summands $\left(X_{i}\right)_{i \geq 1}$ follow a Burr distribution (which constitutes another family of regularly varying distributions with $\bar{F}(x)=\left(1+x^{\gamma} / \theta\right)^{-\alpha}$, $x>0$ ), then one can extend the above described procedure and obtain the complete asymptotic expansion of $\bar{G}(u)$ under quite mild assumptions on the distribution of $N$. After establishing some properties of the Laplace transform of a Burr distribution in Section 2, we derive the asymptotic expansion of $\bar{G}(u)$ in Section 3. In Section 4 we consider the case of a deterministic $N$ and show that then for $u>N$ the asymptotic expansion converges and is equal to $\bar{G}(u)$. In Section 5 , we provide some numerical illustrations and Section 6 contains some final remarks.

\section{A series expansion for the Laplace transform of the Burr distribution}

Consider the Burr distribution with $\bar{F}(x)=\left(1+(x / \theta)^{\gamma}\right)^{-\alpha}$. W.l.o.g. we choose the scale parameter $\theta=1$. Assume further that

$$
h(n):=\frac{1}{\inf _{k \geq 0, n+1-(k+\alpha) \gamma \neq 0}|n+1-(k+\alpha) \gamma|}=\mathcal{O}\left(n^{d}\right)
$$

for some $d>0$ (where $k$ and $n$ are integers) and note that this condition is always fulfilled if $\alpha$ and $\gamma$ are rational numbers. We will now provide a series expansion for $\hat{L}_{\bar{F}}(s)$ that is valid on $\mathbb{D}:=\mathbb{C} \backslash(-\infty, 0]$.

For the Laplace transform of the tail $\bar{F}$ we have

$$
\hat{L}_{\bar{F}}(s)=\int_{0}^{\infty} e^{-s x}\left(1+x^{\gamma}\right)^{-\alpha} \mathrm{d} x=\int_{0}^{R} e^{-s x}\left(1+x^{\gamma}\right)^{-\alpha} \mathrm{d} x+\int_{R}^{\infty} e^{-s x}\left(1+x^{\gamma}\right)^{-\alpha} \mathrm{d} x .
$$

Note that

$$
\int_{0}^{R} e^{-s x}\left(1+x^{\gamma}\right)^{-\alpha} \mathrm{d} x=\sum_{n=0}^{\infty} \frac{(-s)^{n}}{n !} \int_{0}^{R} x^{n}\left(1+x^{\gamma}\right)^{-\alpha} \mathrm{d} x .
$$

Denote with $E_{n}(x):=\int_{1}^{\infty} e^{-x t} / t^{n} \mathrm{~d} t$ the exponential integral, with $\Gamma(\alpha)$ the Gamma function and with $\gamma^{*}(a, x):=x^{-a} / \Gamma(a)\left(\Gamma(a)-\int_{x}^{\infty} e^{-t} t^{a-1} \mathrm{~d} t\right)$ the incomplete Gamma function (cf. (Abramowitz and Stegun, 1964, Equation 6.5.4)). For $R>1$ we have 


$$
\begin{aligned}
\int_{R}^{\infty} & e^{-s x}\left(1+x^{\gamma}\right)^{-\alpha} \mathrm{d} x=\sum_{n=0}^{\infty} \frac{(-1)^{n} \Gamma(\alpha+n)}{\Gamma(\alpha) \Gamma(n+1)} \int_{R}^{\infty} e^{-s x} x^{-(n+\alpha) \gamma} \mathrm{d} x \\
= & \sum_{n=0}^{\infty} \frac{(-1)^{n} s^{(n+\alpha) \gamma-1} \Gamma(\alpha+n)}{\Gamma(\alpha) \Gamma(n+1)} \int_{s R}^{\infty} e^{-x} x^{-(n+\alpha) \gamma} \mathrm{d} x \\
= & \sum_{n=0}^{\infty} \frac{(-1)^{n} s^{(n+\alpha) \gamma-1} \Gamma(\alpha+n)}{\Gamma(\alpha) \Gamma(n+1)} \Gamma(1-(n+\alpha) \gamma, s R) \\
= & \sum_{n=0,(n+\alpha) \gamma \notin \mathbb{N}}^{\infty} \frac{(-1)^{n} s^{(n+\alpha) \gamma-1} \Gamma(\alpha+n)}{\Gamma(\alpha) \Gamma(n+1)} \Gamma(1-(n+\alpha) \gamma) \\
& -\sum_{n=0,(n+\alpha) \gamma \notin \mathbb{N}}^{\infty} \frac{(-1)^{n} \Gamma(\alpha+n)}{\Gamma(\alpha) \Gamma(n+1)} \gamma^{*}(1-(n+\alpha) \gamma, R s) \Gamma(1-(n+\alpha) \gamma) R^{1-(n+\alpha) \gamma} \\
& +\sum_{n=0,(n+\alpha) \gamma \in \mathbb{N}} \frac{(-1)^{n} s^{(n+\alpha) \gamma-1} \Gamma(\alpha+n)}{\Gamma(\alpha) \Gamma(n+1)}(s R)^{1-(n+\alpha) \gamma} E_{(n+\alpha) \gamma}(s R) .
\end{aligned}
$$

Define $\psi(n):=\Gamma^{\prime}(n) / \Gamma(n)$. It then follows that

$$
\begin{gathered}
\int_{R}^{\infty} e^{-s x}\left(1+x^{\gamma}\right)^{-\alpha} \mathrm{d} x=\sum_{n=0,(n+\alpha) \gamma \notin \mathbb{N}}^{\infty} \frac{(-1)^{n} s^{(n+\alpha) \gamma-1} \Gamma(\alpha+n)}{\Gamma(\alpha) \Gamma(n+1)} \Gamma(1-(n+\alpha) \gamma) \\
-\sum_{n=0,(n+\alpha) \gamma \notin \mathbb{N}}^{\infty} \frac{(-1)^{n} \Gamma(\alpha+n)}{\Gamma(\alpha) \Gamma(n+1)} \sum_{k=0}^{\infty} \frac{(-s)^{k}}{(k+1-(n+\alpha) \gamma) k !} R^{k+1-(n+\alpha) \gamma} \\
+\sum_{n=0,(n+\alpha) \gamma \in \mathbb{N}} \frac{(-1)^{n} \Gamma(\alpha+n)}{\Gamma(\alpha) \Gamma(n+1)}\left(\frac{(-s)^{(n+\alpha) \gamma-1}}{\Gamma((n+\alpha) \gamma)}(-\log (s R)+\psi((n+\alpha) \gamma))\right. \\
\left.\quad-\sum_{k=0, k \neq(n+\alpha) \gamma-1}^{\infty} \frac{(-s)^{k}}{(k+1-(n+\alpha) \gamma) k !} R^{k+1-(n+\alpha) \gamma}\right) .
\end{gathered}
$$

From $h(n)=\mathcal{O}\left(n^{d}\right)$ it follows that for $R>1$ the above sums are absolutely convergent. For $|s|<\infty$ we have 


$$
\begin{aligned}
& \int_{0}^{\infty} e^{-s x}\left(1+x^{\gamma}\right)^{-\alpha} \mathrm{d} x=s^{\alpha \gamma-1} \sum_{n=0,(n+\alpha) \gamma \notin \mathbb{N}}^{\infty} \frac{(-1)^{n} \Gamma(\alpha+n) \Gamma(1-(n+\alpha) \gamma)}{\Gamma(\alpha) \Gamma(n+1)} s^{n \gamma} \\
& +s^{\alpha \gamma-1} \sum_{n=0,(n+\alpha) \gamma \in \mathbb{N}}^{\infty} \frac{(-1)^{n+(n+\alpha) \gamma-1} \Gamma(\alpha+n)}{\Gamma(\alpha) \Gamma(n+1) \Gamma((n+\alpha) \gamma)} s^{n \gamma}(\psi((n+\alpha) \gamma)-\log (s)) \\
& +\sum_{n=0}^{\infty} \frac{(-s)^{n}}{n !}\left(\int_{0}^{R} x^{n}\left(1+x^{\gamma}\right)^{-\alpha} \mathrm{d} x\right. \\
& \quad-\sum_{k=0, k \neq \frac{n+1}{\gamma}-\alpha}^{\infty} \frac{(-1)^{k} \Gamma(\alpha+k)}{\Gamma(\alpha) \Gamma(k+1)(n+1-(k+\alpha) \gamma)} R^{n+1-(k+\alpha) \gamma} \\
& \left.-\frac{(-1)^{\frac{n+1}{\gamma}-\alpha} \Gamma\left(\frac{n+1}{\gamma}\right)}{\Gamma(\alpha) \Gamma\left(\frac{n+1}{\gamma}-\alpha+1\right)} \log (R) I_{\left\{\frac{n+1}{\gamma}-\alpha \in \mathbb{N}\right\}}\right) .
\end{aligned}
$$

Dominated convergence and a complex mean value theorem (see Evard and Jafari (1992)) then yield that the function

$g(R, n):=\int_{0}^{R} x^{n}\left(1+x^{\gamma}\right)^{-\alpha} \mathrm{d} x-\sum_{k=0, k \neq \frac{n+1}{\gamma}-\alpha}^{\infty} \frac{(-1)^{k} \Gamma(\alpha+k)}{\Gamma(\alpha) \Gamma(k+1)(n+1-(k+\alpha) \gamma)} R^{n+1-(k+\alpha) \gamma}$


computation of the derivative shows that, for $\inf _{k \in \mathbb{N}}|n+1-(k+\alpha) \gamma|>0, g(R, n)$ is in fact constant in $R>1$. Consequently, for $-1<n<\alpha \gamma-1$, we get by letting $R \rightarrow \infty$

$$
g(R, n)=g(\infty, n)=\int_{0}^{\infty} x^{n}\left(1+x^{\gamma}\right)^{-\alpha} \mathrm{d} x=\frac{1}{n+1} \mathbb{E}\left[X^{n+1}\right]=\frac{\Gamma\left(\frac{n+1}{\gamma}\right) \Gamma\left(\frac{\alpha \gamma-(n+1)}{\gamma}\right)}{\gamma \Gamma(\alpha)} .
$$

By analytic continuation in $n$ we can drop the restriction $-1<n<\alpha \gamma-1$ on $n$ and get for $\inf _{k \in \mathbb{N}}|n+1-(k+\alpha) \gamma|>0$

$$
g(R, n):=\frac{\Gamma\left(\frac{n+1}{\gamma}\right) \Gamma\left(\frac{\alpha \gamma-(n+1)}{\gamma}\right)}{\gamma \Gamma(\alpha)} .
$$

This means that if we consider $\mathbb{E}\left[X^{n+1}\right]$ as a function in $n(-1<n<\alpha \gamma-1)$, then the analytic continuation of this function is given by $(n+1) g(R, n)$. So it makes sense to define $\mathbb{E}\left[X^{n}\right]:=n g(R, n-1)$, which for values of $n$ with $-1<n<\alpha \gamma-1$ coincides with the usual definition of $\mathbb{E}\left[X^{n}\right]$, but now can be evaluated for $n>\alpha \gamma-1$. At last we have to discuss the case of $n=n_{0}$, when there exists a $k_{0} \in \mathbb{N}$ with $k_{0}=\left(n_{0}+1\right) / \gamma-\alpha$. We 
have

$$
\begin{aligned}
g\left(R, n_{0}\right) & =\lim _{n \rightarrow n_{0}} g(R, n)+\frac{(-1)^{k_{0}} \Gamma\left(\alpha+k_{0}\right)}{\Gamma(\alpha) \Gamma\left(k_{0}+1\right)\left(n+1-\left(k_{0}+\alpha\right) \gamma\right)} R^{n+1-\left(k_{0}+\alpha\right) \gamma} \\
& =\lim _{n \rightarrow n_{0}} \frac{\Gamma\left(\frac{n+1}{\gamma}\right) \Gamma\left(-k_{0}-\frac{n-n_{0}}{\gamma}\right)}{\Gamma(\alpha) \gamma}+\frac{(-1)^{k_{0}} \Gamma\left(\frac{n_{0}+1}{\gamma}\right)}{\Gamma(\alpha) \Gamma\left(k_{0}+1\right)\left(n-n_{0}\right)} R^{n-n_{0}} \\
& =\frac{(-1)^{\frac{n_{0}+1}{\gamma}-\alpha+1} \Gamma\left(\frac{n_{0}+1}{\gamma}\right)}{\gamma \Gamma(\alpha) \Gamma\left(\frac{n_{0}+1}{\gamma}-\alpha+1\right)}\left(\psi\left(\frac{n_{0}+1}{\gamma}\right)-\psi\left(\frac{n_{0}+1}{\gamma}-\alpha+1\right)-\gamma \log (R)\right)
\end{aligned}
$$

from which it follows that

$$
\begin{aligned}
\hat{L}_{\bar{F}}(s)= & s^{\alpha \gamma-1} \sum_{n=0,(n+\alpha) \gamma \notin \mathbb{N}}^{\infty} \frac{(-1)^{n} \Gamma(\alpha+n) \Gamma(1-(n+\alpha) \gamma)}{\Gamma(\alpha) \Gamma(n+1)} s^{n \gamma} \\
& +s^{\alpha \gamma-1} \sum_{n=0,(n+\alpha) \gamma \in \mathbb{N}}^{\infty} \frac{(-1)^{n+(n+\alpha) \gamma-1} \Gamma(\alpha+n)}{\Gamma(\alpha) \Gamma(n+1) \Gamma((n+\alpha) \gamma)} s^{n \gamma}(\psi((n+\alpha) \gamma)-\log (s)) \\
& +\sum_{n=0, \frac{n+1}{\gamma}-\alpha \notin \mathbb{N}}^{\infty} \frac{(-s)^{n}}{n !} \frac{\Gamma\left(\frac{n+1}{\gamma}\right) \Gamma\left(\frac{\alpha \gamma-(n+1)}{\gamma}\right)}{\gamma \Gamma(\alpha)} \\
& +\sum_{n=0, \frac{n+1}{\gamma}-\alpha \in \mathbb{N}}^{\infty} \frac{(-s)^{n}}{n !} \frac{(-1)^{\frac{n+1}{\gamma}-\alpha+1} \Gamma\left(\frac{n+1}{\gamma}\right)}{\gamma \Gamma(\alpha) \Gamma\left(\frac{n+1}{\gamma}-\alpha+1\right)}\left(\psi\left(\frac{n+1}{\gamma}\right)-\psi\left(\frac{n+1}{\gamma}-\alpha+1\right)\right) .
\end{aligned}
$$

On the other hand, one can obtain an asymptotic expansion for $\hat{L}_{f}(s)$ for $|s| \rightarrow \infty$ and $\operatorname{Re}(s)>-t_{0}$ for a $t_{0}>0$. If $|\gamma \phi|<\pi$, then we have that for $|\arg (s)-\phi|<\pi / 2$ (cf. Olver $(1997))$

$$
\hat{L}_{f}(s):=\int_{0}^{\infty} e^{-s x} f(x) \mathrm{d} x=e^{-\iota \phi} \int_{0}^{\infty} e^{-s e^{-\iota \phi} x} f\left(x e^{-\iota \phi}\right) \mathrm{d} x .
$$

By an application of Watson's Lemma (see e.g. Olver (1997)), we arrive at

$$
\hat{L}_{\bar{F}}(s):=e^{-\iota \phi} \int_{0}^{\infty} e^{-s e^{-\iota \phi} x} \bar{F}\left(x e^{-\iota \phi}\right) \mathrm{d} x \sim \sum_{n=0}^{\infty} \frac{(-1)^{n} \Gamma(\alpha+n) \Gamma(\gamma n+1)}{\Gamma(\alpha) n !} s^{-(n \gamma+1)} .
$$

\section{Complete asymptotic expansion of the tail of a compound sum with Burr distributed random variables}

Denote the probability generating function of the number $N$ of summands by $Q_{N}(z):=$ $\mathbb{E}\left[z^{N}\right]$, then

$$
\hat{L}_{\bar{G}}(s)=\frac{1}{s}-\frac{1}{s} Q_{N}\left(\hat{L}_{f}(s)\right),
$$

and for an $s_{0}>0$ we get

$$
\bar{G}(u)=1-\frac{1}{2 \pi \iota} \int_{s_{0}-\iota \infty}^{s_{0}+\iota \infty} e^{u s} \frac{1}{s} Q_{N}\left(\hat{L}_{f}(s)\right) \mathrm{d} s,
$$


where $\hat{L}_{f}(s)$ denotes the Laplace transform of the probability density function of the $X_{i}$. At first we consider the case that $n+1-(k+\alpha) \gamma \notin \mathbb{N}$ for all $k \geq 0$ and $n \geq 0$. Due to (4), we can apply Lemma 2.9 of Albrecher and Kortschak (2009) to

$$
g(s):=Q_{N}\left(\hat{L}_{f}(s)\right)
$$

to obtain the following result (see also Theorem 4.1 of Albrecher and Kortschak (2009)).

Proposition 3.1. Let $X_{1}, X_{2}, \ldots$ be independent and identically distributed Burr random variables with distribution tail $\bar{F}(x)=\left(1+x^{\gamma}\right)^{-\alpha}$ and let $N$ be an integer-valued random variable independent of $X_{i}$ such that there exists an $\epsilon>0$ with

$$
\mathbb{E}\left[(1+\epsilon)^{N}\right]=\sum_{n=0}^{\infty} \mathbb{P}(N=n)(1+\epsilon)^{n}<\infty
$$

Then there exists a $t_{0}>0$ with

$$
\bar{G}(u) \approx-\frac{1}{\pi} \int_{0}^{t_{0}} \frac{1}{x} e^{-u x} \operatorname{Im}\left(Q_{N}\left(\hat{L}_{f}(-x)\right)\right) \mathrm{d} x,
$$

where for two functions $a(x)$ and $b(x)$ the symbol $a(x) \approx b(x)$ means that there exists $a$ $\delta>0$ with $a(x)=b(x)+\mathcal{O}\left(e^{-\delta x}\right)$.

This result can now be used to get an asymptotic expansion for $\bar{G}(u)$. At first, write

$$
Q_{N}(z)=\sum_{n=0}^{\infty} q_{n}(z-1)^{n}
$$

Note that for $n>0$

$$
q_{n}=\left.\frac{1}{n !} \frac{\partial Q_{N}(z)}{\partial(z)}\right|_{z=1}=\frac{\mathbb{E}[N(N-1) \cdots(N-n+1)]}{n !} .
$$

Further we have from (3)

$$
\hat{L}_{f}(s)=1-s^{\alpha \gamma} \sum_{n=0}^{\infty} \frac{(-1)^{n} \Gamma(\alpha+n) \Gamma(1-(n+\alpha) \gamma)}{\Gamma(\alpha) \Gamma(n+1)} s^{n \gamma}+\sum_{n=1}^{\infty} \frac{(-s)^{n}}{n !} \mathbb{E}\left[X^{n}\right],
$$

where for $n>\alpha \gamma$ we used the analytic continuation of $\mathbb{E}\left[X^{n}\right]$ defined by equations (1) and (2). Define $a_{n}^{m}$ such that

$$
\left(\sum_{n=1}^{\infty} \frac{(-s)^{n}}{n !} \mathbb{E}\left[X^{n}\right]\right)^{m}=\sum_{n=m}^{\infty} a_{n}^{m}(-s)^{n}
$$

and $b_{n}^{m}$ such that

$$
\left(-s^{\alpha \gamma} \sum_{n=0}^{\infty} \frac{(-1)^{n} \Gamma(\alpha+n) \Gamma(1-(n+\alpha) \gamma)}{\Gamma(\alpha) \Gamma(n+1)} s^{n \gamma}\right)^{m}=\sum_{n=0}^{\infty} b_{n}^{m} s^{(n+m \alpha) \gamma} .
$$


It follows that

$$
\left(\hat{L}_{f}(-s)-1\right)^{m}=\sum_{k=1}^{m}\left(\begin{array}{c}
m \\
k
\end{array}\right) \sum_{l=0}^{\infty} \sum_{n=m-k}^{\infty} a_{n}^{m-k} b_{l}^{k} s^{(l+k \alpha) \gamma+n} e^{\iota \pi(l+k \alpha) \gamma} .
$$

We have that for $s$ in the neighborhood of 0 :

$$
\operatorname{Im}\left(Q_{N}\left(\hat{L}_{f}(-s)\right)\right)=\sum_{m=0}^{\infty} q_{m} \sum_{k=1}^{m}\left(\begin{array}{c}
m \\
k
\end{array}\right) \sum_{l=0}^{\infty} \sum_{n=m-k}^{\infty} a_{n}^{m-k} b_{l}^{k} s^{(l+k \alpha) \gamma+n} \sin (\pi(l+k \alpha) \gamma) .
$$

Inserting this formula into the integral of Proposition 3.1, another application of Watson's Lemma (e.g. Olver (1997)) yields

$$
\begin{aligned}
& \int_{0}^{t_{0}} \frac{1}{x} e^{-u x} \operatorname{Im}\left(Q_{N}\left(\hat{L}_{f}(-x)\right)\right) \mathrm{d} x \\
& \sim \sum_{m=0}^{\infty} q_{m} \sum_{k=1}^{m}\left(\begin{array}{c}
m \\
k
\end{array}\right) \sum_{l=0}^{\infty} \sum_{n=m-k}^{\infty} a_{n}^{m-k} b_{l}^{k} \sin (\pi(l+k \alpha) \gamma) u^{-((l+k \alpha) \gamma+n)} \Gamma((l+k \alpha) \gamma+n) \\
& \sim \sum_{l, n \geq 0, k \geq 1} u^{-((l+k \alpha) \gamma+n)} b_{l}^{k} \sin (\pi(l+k \alpha) \gamma) \Gamma((l+k \alpha) \gamma+n) \sum_{m=k}^{k+n} a_{n}^{m-k} q_{m}\left(\begin{array}{c}
m \\
k
\end{array}\right) .
\end{aligned}
$$

Note that for $n<\alpha \gamma$

$$
\sum_{m=k}^{k+n} a_{n}^{m-k} q_{m}\left(\begin{array}{c}
m \\
k
\end{array}\right)=\frac{\mathbb{E}\left[N(N-1) \cdots(N-k+1) S_{N-k}^{n}\right]}{k ! n !},
$$

and for $k=1, u>1$ we get

$$
-\sum_{l=0}^{\infty} u^{-((l+k \alpha) \gamma+n)} b_{l}^{1} \sin (\pi(l+\alpha) \gamma) \Gamma((l+\alpha) \gamma+n)=\pi(-1)^{n} \bar{F}^{(n)}(u),
$$

where $\bar{F}^{(n)}(u)$ is the $n$-th derivative of $\bar{F}(u)$.

By equation (6) we have, up to a constant, provided an asymptotic expansion for $\bar{G}(u)$ if there exist no positive integers $k$ and $n$ with $n+1-(k+\alpha) \gamma \in \mathbb{N}$. In case there exist positive integers $k$ and $n$ with $n+1-(k+\alpha) \gamma \in \mathbb{N}$, we can find real constants $a_{n}, b_{n}$, and $c_{n}$ such that (3) reads:

$$
\hat{L}_{f}(-s)-1=\sum_{n=1}^{\infty} a_{n} s^{n}+\sum_{n=0}^{\infty} b_{n} s^{(n+\alpha) \gamma} e^{\iota \pi(n+\alpha) \gamma}+\sum_{n=1}^{\infty} c_{n} s^{(n+\alpha) \gamma}(\log (s)+\iota \pi) .
$$

Define $a_{n}^{i}, b_{n}^{i}$ and $c_{n}^{i}$ analogously as above and define

$$
C_{l, k, n, h}:=\sum_{j=0}^{k-h} \sum_{i=0}^{l} \sum_{m=k}^{n+k}\left(\begin{array}{c}
m \\
k, j, h
\end{array}\right) \sin \left(\pi(i+j \alpha) \gamma+\frac{\pi}{2}(k-j-h)\right) \pi^{k-j-h} q_{m} a_{n}^{m-k} b_{i}^{j} c_{l-i}^{k-j} .
$$

After some algebraic manipulations one gets

$$
\operatorname{Im}\left(Q_{N}\left(\hat{L}_{f}(-s)\right)\right)=\sum_{l, n \geq 0, k \geq 1} \sum_{h=0}^{k-1} C_{l, k, n, h}(\log (s))^{h} s^{(l+k \alpha) \gamma+n} .
$$


Define finally

$$
\begin{aligned}
\bar{C}(m, n) & :=\int_{0}^{\infty} e^{-x} \log (x)^{n} x^{m-1} \mathrm{~d} x \text { and } \\
\hat{C}_{l, k, n, h} & :=-\frac{1}{\pi} \sum_{m=h}^{k-1}\left(\begin{array}{c}
m \\
h
\end{array}\right)(-1)^{h} C_{l, k, n, m} \bar{C}((l+k \alpha) \gamma+n, m-h) .
\end{aligned}
$$

It then follows (analogously to the case $n+1-(k+\alpha) \gamma \notin \mathbb{N}$ for all positive integers $k$ and $n$ ) that

$$
\bar{G}(u) \sim \sum_{l, n \geq 0, k \geq 1} \sum_{h=0}^{k-1} \hat{C}_{l, k, n, h}(\log (u))^{h} u^{-(l+k \alpha) \gamma-n} .
$$

\section{The case of deterministic $N$}

In this section we will assume that $N$ is deterministic. We will only consider the case that $n+1-(k+\alpha) \gamma \notin \mathbb{N}$ for all $k \geq 0$ and $n \geq 0$. We will show that then the series (6) converges for $u>N$. At first note that for deterministic $N$

$$
q_{m}=\left(\begin{array}{l}
N \\
m
\end{array}\right)
$$

so that $q_{m}=0$ for $m>N$. Consequently the sum over the $k$ in (6) is finite and hence we can consider a fixed $1 \leq k \leq N$. For all $\epsilon>0$ there exists a constant $C_{1}$ such that for all $n \geq 0$

$$
\left|a_{n}^{1}\right|=\frac{1}{n !}\left|\frac{n \pi \Gamma\left(\frac{n}{\gamma}\right)}{\gamma \Gamma(\alpha) \sin \left(\pi\left(\alpha-\frac{n}{\gamma}\right)\right) \Gamma\left(\frac{n}{\gamma}-\alpha\right)\left(\frac{n}{\gamma}-\alpha\right)}\right| \leq C_{1} \frac{(1+\epsilon)^{n}}{n !}
$$

(note that we have assumed that $h(n)=\mathcal{O}\left(n^{d}\right)$ ). It follows that $\left|a_{n}^{m}\right| \leq C_{1}^{m}((1+\epsilon) m)^{n} / n$ !. Hence there exists a constant $C_{2}$ such that for all $n$

$$
\sum_{m=k}^{k+n}\left|a_{n}^{m-k} q_{m}\left(\begin{array}{c}
m \\
k
\end{array}\right)\right| \leq C_{2} \frac{((1+\epsilon)(N-k))^{n}}{n !} .
$$

Since for $x<1$ and $c>0$

$$
\sum_{n=0}^{\infty} \frac{\Gamma(c+n) x^{n}}{n !}(1-x)^{-c} \Gamma(c),
$$

we get that for $u>(1+\epsilon)(N-k)$ and $l \in \mathbb{N}_{0}$

$$
\begin{aligned}
\sum_{n=0}^{\infty} \mid u^{-n} \sin (\pi(l+k \alpha) \gamma) \Gamma((l+k \alpha) \gamma & +n)\left|\sum_{m=k}^{k+n}\right| a_{n}^{m-k} q_{m}\left(\begin{array}{c}
m \\
k
\end{array}\right) \mid \\
& \leq C_{2} \Gamma((l+k \alpha) \gamma)\left(\frac{u}{u-(1+\epsilon)(N-k)}\right)^{(l+k \alpha) \gamma}
\end{aligned}
$$


It is left to show the convergence for

$$
\sum_{l=0}^{\infty}\left|b_{l}^{k}\right| \Gamma((l+k \alpha) \gamma)\left(\frac{1}{u-(1+\epsilon)(N-k)}\right)^{(l+k \alpha) \gamma} .
$$

Note that for all $\epsilon>0$ there exists a constant $C_{3}$ such that for all $l \in \mathbb{N}_{0}$

$$
\left|b_{l}^{1}\right|=\left|\frac{\pi \Gamma(\alpha+l)}{\Gamma((l+\alpha) \gamma) \sin ((l+\alpha) \gamma) \Gamma(\alpha) \Gamma(l+1)}\right| \leq C_{3} \frac{(1+\epsilon)^{\gamma l}}{\Gamma((l+\alpha) \gamma)} .
$$

We want to show that $\left|b_{l}^{k}\right| \leq C_{4}(l+1)^{k-1}((1+\epsilon) k)^{\gamma l} / \Gamma((l+\alpha) \gamma)$. For that purpose define $\hat{b}_{l}^{k}$ such that

$$
\left(\sum_{l=0}^{\infty} \frac{s^{l}}{\Gamma((l+\alpha) \gamma)}\right)^{k}=\sum_{l=0}^{\infty} \hat{b}_{l}^{k} s^{l} .
$$

Obviously $\left|b_{l}^{k}\right| \leq C_{3}^{k}(1+\epsilon)^{\gamma l} \hat{b}_{l}^{k}$ and we have to show that $\hat{b}_{l}^{k} \leq C_{5}^{k-1}(l+1)^{k-1} k^{\gamma l} / \Gamma((l+\alpha) \gamma)$. At first note that for all $1 \leq m \leq l-1$ there exists a constant $C_{5}>1 / \Gamma(\alpha \gamma)$ such that

$$
\frac{\Gamma((l+\alpha) \gamma)}{\Gamma((m+\alpha) \gamma) \Gamma((l-m+\alpha) \gamma)} \leq C_{5} \frac{l^{\gamma l}}{m^{m \gamma}(l-m)^{(l-m) \gamma}}
$$

Further note that

$$
\frac{l^{\gamma l}}{k^{\gamma m} m^{m \gamma}(l-m)^{(l-m) \gamma}} \leq\left(\frac{k+1}{k}\right)^{l \gamma} .
$$

We use induction over $k$ to prove $\hat{b}_{l}^{k} \leq C_{5}^{k-1}(l+1)^{k-1} k^{\gamma l} / \Gamma((l+\alpha) \gamma)$. This assertion is true for $k=1$. Assume further that it holds for a given $k$. Then, indeed for $k+1$

$$
\begin{aligned}
\frac{\hat{b}_{l}^{k+1} \Gamma((l+\alpha) \gamma)}{C_{5}^{k}(l+1)^{k}(k+1)^{\gamma l}} & =\frac{\Gamma((l+\alpha) \gamma)}{C_{5}^{k}(l+1)^{k}(k+1)^{\gamma l}} \sum_{m=0}^{l} \frac{\hat{b}_{l-m}^{k}}{\Gamma((m+\alpha) \gamma)} \\
& \leq \frac{\Gamma((l+\alpha) \gamma) k^{\gamma l}}{C_{5}^{k}(l+1)^{k}(k+1)^{\gamma l}} \sum_{m=0}^{l} \frac{C_{5}^{k-1}(l+1-m)^{k-1}}{k^{\gamma m} \Gamma((m+\alpha) \gamma) \Gamma((l-m+\alpha) \gamma)} \\
& \leq \frac{k^{\gamma l}}{C_{5}(l+1)(k+1)^{\gamma l}} \sum_{m=0}^{l} \frac{\Gamma((l+\alpha) \gamma)}{k^{\gamma m} \Gamma((m+\alpha) \gamma) \Gamma((l-m+\alpha) \gamma)} \\
& \leq \frac{k^{\gamma l}}{C_{5}(l+1)(k+1)^{\gamma l}} \sum_{m=0}^{l} C_{5}\left(\frac{k+1}{k}\right)^{l \gamma}=1 .
\end{aligned}
$$

We now also get that $\left|b_{l}^{k}\right| \leq C_{4}(l+1)^{k-1}((1+\epsilon) k)^{\gamma l} / \Gamma((l+\alpha) \gamma)$. It follows from (8) that (6) converges if

$$
\frac{(1+\epsilon) k}{u-(1+\epsilon)(N-k)}<1 \quad \text { or equivalently } \quad u>(1+\epsilon) N
$$

Since $\epsilon>0$ was arbitrary we get that the sum in (6) converges for all $u>N$ and hence

$$
\begin{aligned}
\int_{0}^{\infty} \frac{1}{x} e^{-u x} \operatorname{Im}\left(Q_{N}\left(\hat{L}_{f}(-x)\right)\right) \mathrm{d} x & \\
& =\sum_{l, n \geq 0, k \geq 1} u^{-((l+k \alpha) \gamma+n)} b_{l}^{k} \sin (\pi(l+k \alpha) \gamma) \Gamma((l+k \alpha) \gamma+n) \sum_{m=k}^{k+n} a_{n}^{m-k} q_{m}\left(\begin{array}{c}
m \\
k
\end{array}\right) .
\end{aligned}
$$


Now it remains to show that for $u>N$ ( $N$ deterministic) we do not only have the approximation but the equality

$$
\begin{aligned}
-\frac{1}{\pi} \int_{0}^{\infty} \frac{1}{x} e^{-u x} \operatorname{Im}\left(Q_{N}\left(\hat{L}_{f}(-x)\right)\right) \mathrm{d} x & \\
& =-\frac{1}{\pi} \int_{0}^{\infty} \frac{1}{x} e^{-u x} \operatorname{Im}\left(\left(\hat{L}_{f}(-x)\right)^{N}\right) \mathrm{d} x=\mathbb{P}\left(S_{N}>u\right) .
\end{aligned}
$$

From equation (7) we get that this equality holds for $N=1$. It follows that

$$
\hat{L}_{f}(s)=\int_{0}^{1} e^{-s x} f(x) \mathrm{d} x-\frac{e^{-s}}{\pi} \int_{0}^{\infty} \frac{1}{s+x} e^{-x} \operatorname{Im}\left(\hat{L}_{f}(-x)\right) \mathrm{d} x .
$$

From this we can deduce that for $\epsilon>0, e^{s N+\epsilon} \frac{1}{s} \hat{L}_{f}(s)^{N} \rightarrow 0$ for $\operatorname{Re}(s)$ bounded from above and $|s| \rightarrow 0$. Note that for $u>N, 0<\epsilon<u-N$ and $s_{0}>0$

$$
\mathbb{P}\left(S_{N}>u\right)=\frac{1}{2 \pi \iota} \int_{s_{0}-\iota \infty}^{s_{0}+\iota \infty} e^{(u-(N+\epsilon)) s} e^{(N+\epsilon) s}\left(\frac{1}{s}-\frac{1}{s} \hat{L}_{f}(s)^{N}\right) \mathrm{d} s .
$$

Hence we can use the same contour integration as in Brennan et al. (1968) to establish that (9) holds.

\section{A numerical illustration}

Let us choose $N$ to be Poisson distributed with parameter $\lambda=10$ and choose $\alpha=0.7$ and $\gamma=2.3$ for the parameters of the Burr distribution. In this case the first moment exists, but the second moment does not exist. At first we provide three plots for the evaluation of $\hat{L}_{f}(s)$ for the Burr distribution. In Figure 1 we see the imaginary and real part of $\hat{L}_{f}(s)$ for $s$ with $-5<\operatorname{Re}(s)<5$ and $-5<\operatorname{Im}(s)<5$. One can see the branch cut of $\hat{L}_{f}(s)$ on the negative real axis. Figure 2 depicts the relative error of $\hat{L}_{f}(s)$, when using the series expansion of equation (3). For the chosen set of parameters, one in fact only has to evaluate two sums. One should note that the absolute value of these sums tends to $\infty$ when $|s| \rightarrow \infty$. Since $\lim _{\operatorname{Re}(s) \rightarrow \infty} \hat{L}_{f}(s)=0$, this is numerically not a favorable situation. Let us turn to the asymptotic expansion. Since $\inf _{k \geq 0, n+1-(k+\alpha) \gamma \neq 0}|n+1-(k+\alpha) \gamma|>$ 0 , the asymptotic expansion is, up to a constant, given by (6). This expansion is not convergent, so one can only use finitely many terms of the expansion for an asymptotic approximation. As usual in such a situation, it is not so clear how many terms to take for a useful approximation. Although there is no precise answer to this question, one can state the following: In Section 4 we have seen that if one drops all terms with $m>p$, then one obtains a convergent sum. We will use these ideas to get two types of asymptotic approximations. In the first case we will sum only over those values of $n$ and $k$ with $n+k \leq p$. Bounding 1 by $p$, this then leads to the approximation

$$
\begin{aligned}
a_{p}(u):=-\frac{1}{\pi} \sum_{l=0}^{p} \sum_{k=1}^{p} \sum_{n=0}^{p-k} u^{-((l+k \alpha) \gamma+n)} b_{l}^{k} & \\
& \times \sin (\pi(l+k \alpha) \gamma) \Gamma((l+k \alpha) \gamma+n) \sum_{m=k}^{k+n} a_{n}^{m-k} q_{m}\left(\begin{array}{c}
m \\
k
\end{array}\right) .
\end{aligned}
$$





Figure 1: A plot of the real and imaginary part, respectively, of $\hat{L}_{f}(s)$ in the complex plane

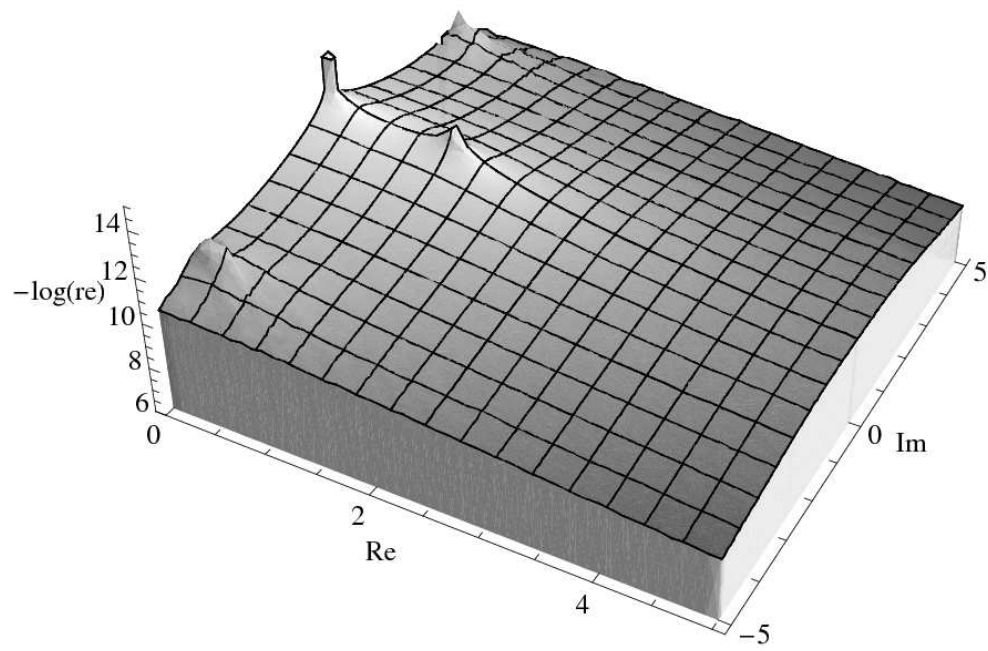

Figure 2: Relative error of the evaluation of the $\hat{L}_{f}(s)$ 


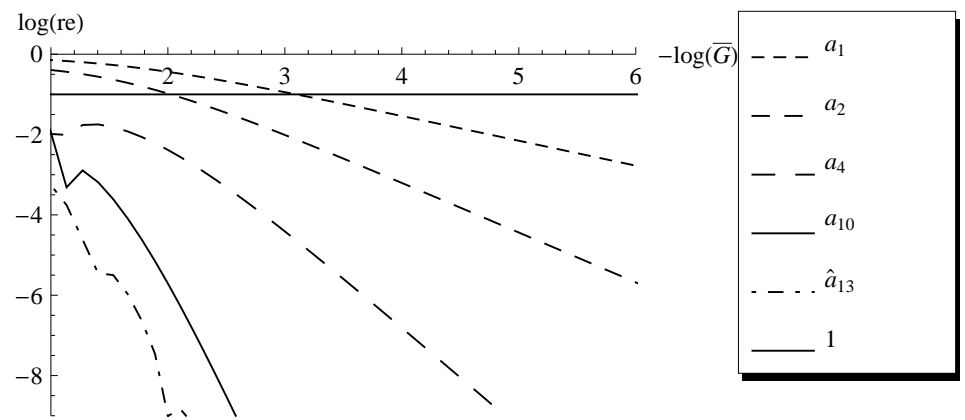

Figure 3: A log-log-plot for the relative error re $=\left|a_{p}(u)-\bar{G}(u)\right| / \bar{G}(u)$ of different asymptotic approximations $a_{p}(u)$ for $\bar{G}(u)$.

As a second choice one can use an approximation that is truncated at $\min (k+n, m)$, so that one gets

$$
\begin{aligned}
\hat{a}_{p}(u):=-\frac{1}{\pi} \sum_{l=0}^{3 p} \sum_{k=1}^{p} \sum_{n=0}^{3 p} u^{-((l+k \alpha) \gamma+n)} b_{l}^{k} & \\
& \quad \times \sin (\pi(l+k \alpha) \gamma) \Gamma((l+k \alpha) \gamma+n) \sum_{m=k}^{\min (k+n, p)} a_{n}^{m-k} q_{m}\left(\begin{array}{c}
m \\
k
\end{array}\right) .
\end{aligned}
$$

Note that the heuristic idea of both approximations is to use $\mathbb{P}\left(S_{N}>u ; N \leq p\right)$ as an approximation for $\mathbb{P}\left(S_{N}>u\right)$, and use an approximation for $\mathbb{P}\left(S_{N}>u ; N \leq p\right)$ which is at the same time an asymptotic approximation for $\mathbb{P}\left(S_{N}>u\right)$ of higher order. In Figure 3 we provide a log-log-plot of the relative error for the approximation of the $\bar{G}(u)$ for different values of $p$. Concretely, on the $x$-axis we plot $-\log _{10}(\bar{G}(u))$ and on the $y$-axis we plot $\log _{10}\left(\left|a_{p}(u)-\bar{G}(u)\right| / \bar{G}(u)\right)$, where $\bar{G}(u)$ is the exact value that we obtained by a sufficiently accurate (and correspondingly time-consuming) numerical inversion of the Laplace transform as described in Abate and Valkó (2004). One can see that in this example, the approximation $a_{4}(u)$ has for all considered values a relative error less than $10 \%$ and $\hat{a}_{13}(u)$ has for all considered values a relative error less than $0.1 \%$. Finally one should note that a higher value of $p$ need not lead to a better approximation for a given $u$. Especially the choice $p>u$ would not make sense since then the underlying sum does not converge any more.

\section{Conclusion}

We derived a series expansion for the Laplace transform of the Burr distribution and subsequently an asymptotic expansion of the tail of a compound sum with Burr distributed summands in terms of $u^{\beta_{i}}$. The proofs exploided the property of the Burr distribution that

$$
\bar{F}(x)=\sum_{n=0}^{\infty} a_{m} x^{-\beta_{m}}
$$


for all $x>R_{0}$ and that the exponents $\beta_{i}$ are sufficiently bounded away from the integers. Furthermore, it was useful that $\mathbb{E}\left[X^{n}\right]$ could be easily extended to all values of $n$ (such an extension was provided by the function $n g(R, n-1)$ for arbitrary $R$; however, in the absence of an explicit expression for this function, its practical use may be limited). In principle, the proposed method will hence also work for the transformed beta distribution with density

$$
f(x):=\frac{\Gamma(\alpha+\tau)}{\Gamma(\alpha) \Gamma(\tau)} \frac{\gamma(x / \theta)^{\gamma \tau}}{x\left(1+(x / \theta)^{\gamma}\right)^{\alpha+\tau}},
$$

which contains the Burr distribution as a special case (cf Klugman et al. (2008)) and for the Fréchet distribution with $F(x)=e^{-x^{-\alpha}}$ (cf. Embrechts et al. (1997)) as well as for their integrated tail distributions and their shifted versions. The challenge then again consists of identifying an appropriate analytic extension of $\mathbb{E}\left[X^{n}\right]$ for all $n$ and establishing properties of the asymptotic expansion of the Laplace transform of the summands along certain complex contours in the vicinity of the imaginary axis.

Acknowledgements. The authors would like to thank Gordon Willmot for stimulating discussions on the topic and an anonymous referee for several helpful suggestions to improve the presentation of the manuscript.

\section{References}

Abate, J., Valkó, P. P., 2004. Multi-precision Laplace transform inversion. International Journal for Numerical Methods in Engineering 60 (5), 979-993.

Abramowitz, M., Stegun, I. A., 1964. Handbook of mathematical functions with formulas, graphs, and mathematical tables. Vol. 55 of National Bureau of Standards Applied Mathematics Series. Washington, D.C.

Albrecher, H., Hipp, C., Kortschak, D., 2010. Higher order expansions for compound distributions and ruin probabilities with subexponential claims. Scand. Actuar. J., to appear.

Albrecher, H., Kortschak, D., 2009. On ruin probability and aggregate claim representations for Pareto claim size distributions. Insurance: Mathematics and Economics 45 (3), $362-373$.

Asmussen, S., 2003. Applied probability and queues, 2nd Edition. Vol. 51 of Applications of Mathematics. Springer-Verlag, New York.

Baltrūnas, A., Omey, E., 1998. The rate of convergence for subexponential distributions. Liet. Mat. Rink. 38 (1), 1-18.

Barbe, P., McCormick, W. P., 2005. Asymptotic expansions of convolutions of regularly varying distributions. J. Aust. Math. Soc. 78 (3), 339-371.

Barbe, P., McCormick, W. P., 2009. Asymptotic expansions for infinite weighted convolutions of heavy tail distributions and applications. American Mathematical Society. 
Barbe, P., McCormick, W. P., Zhang, C., 2007. Asymptotic expansions for distributions of compound sums of random variables with rapidly varying subexponential distribution. J. Appl. Probab. 44 (3), 670-684.

Blum, M., 1970. On the sums of independently distributed Pareto variates. SIAM J. Appl. Math. 19, 191-198.

Borovkov, A. A., Borovkov, K. A., 2002. On probabilities of large deviations for random walks. i. regularly varying distribution tails. Theory of Probability and its Applications $46(2), 193-213$.

Brennan, L., Reed, I., Sollfrey, W., 1968. A comparison of average-likelihood and maximum-likelihood ratio tests for detecting radar targets of unknown Doppler frequency. IEEE Trans. Information Theory 14 (1), 104-110.

Embrechts, P., Klüppelberg, C., Mikosch, T., 1997. Modelling extremal events, for insurance and finance. Vol. 33 of Applications of Mathematics (New York). Springer-Verlag, Berlin.

Embrechts, P., Veraverbeke, N., 1982. Estimates for the probability of ruin with special emphasis on the possibility of large claims. Insurance Math. Econom. 1 (1), 55-72.

Evard, J.-C., Jafari, F., 1992. A complex Rolle's theorem. Amer. Math. Monthly 99 (9), 858-861.

Geluk, J. L., 1992. Second order tail behaviour of a subordinated probability distribution. Stochastic Process. Appl. 40 (2), 325-337.

Geluk, J. L., 1996. Tails of subordinated laws: the regularly varying case. Stochastic Process. Appl. 61 (1), 147-161.

Geluk, J. L., Peng, L., de Vries, C. G., 2000. Convolutions of heavy-tailed random variables and applications to portfolio diversification and MA(1) time series. Adv. in Appl. Probab. 32 (4), 1011-1026.

Grübel, R., 1987. On subordinated distributions and generalized renewal measures. Ann. Probab. 15 (1), 394-415.

Klugman, S. A., Panjer, H. H., Willmot, G. E., 2008. Loss models, 3rd Edition. Wiley Series in Probability and Statistics. John Wiley \& Sons Inc., Hoboken, NJ.

Olver, F. W. J., 1997. Asymptotics and special functions. AKP Classics. A K Peters Ltd., Wellesley, MA.

Rolski, T., Schmidli, H., Schmidt, V., Teugels, J., 1999. Stochastic processes for insurance and finance. Wiley Series in Probability and Statistics. John Wiley \& Sons Ltd., Chichester. 